\title{
Strategy for solving semi-analytically three-dimensional transient flow in a coupled $N$-layer aquifer system
}

\author{
E. J. M. Veling • C. Maas
}

Received: 1 October 2007 / Accepted: 23 October 2008 / Published online: 18 November 2008

(C) The Author(s) 2008. This article is published with open access at Springerlink.com

\begin{abstract}
Efficient strategies for solving semi-analytically the transient groundwater head in a coupled $N$-layer aquifer system $\phi_{i}(r, z, t), i=1, \ldots, N$, with radial symmetry, with full $z$-dependency, and partially penetrating wells are presented. Aquitards are treated as aquifers with their own horizontal and vertical permeabilities. Since the vertical direction is fully taken into account, there is no need to pose the Dupuit assumption, i.e., that the flow is mainly horizontal. To solve this problem, integral transforms will be employed: the Laplace transform for the $t$-variable (with transform parameter $p$ ), the Hankel transform for the $r$-variable (with transform parameter $\alpha$ ) and a particular form of a generalized Fourier transform for the vertical direction $z$ with an infinite set of eigenvalues $\lambda_{m}^{2}$ (with the discrete index $m$ ). It is possible to solve this problem in the form of a semi-analytical solution in the sense that an analytical expression in terms of the variables $r$ and $z$, transform parameter $p$, and eigenvalues $\lambda_{m}^{2}(p)$ of the generalized Fourier transform can be given or in terms of the variables $z$ and $t$, transform parameter $\alpha$, and eigenvalues $\lambda_{m}^{2}(\alpha)$. The calculation of the eigenvalues $\lambda_{m}^{2}$ and the inversion of these transformed solutions can only be done numerically. In this context the application of the generalized Fourier transform is novel. By means of this generalized Fourier transform, transient problems with horizontal symmetries other than radial can be treated as well. The notion of analytical solution versus numerical solution is discussed and a classification of analytical solutions is proposed in seven classes. The expressions found in this paper belong to Class 6 , meaning that the transformed solutions are written in terms of eigenvalues which depend on one transform parameter (here $p$ or $\alpha$ ). Earlier solutions to the transient problem belong to Class 7, where the eigenvalues depend on two transform parameters. The theory is applied to three examples.
\end{abstract}

Keywords Analytical solution · Coupled aquifers · Integral transformation · Transient flow

E. J. M. Veling $(\varangle) \cdot$ C. Maas

Water Resources Section, Faculty of Civil Engineering and Geosciences, Delft University of Technology, P.O. Box 5048, 2600 GA Delft, The Netherlands

e-mail: E.J.M.Veling@TUDelft.nl

C. Maas

KWR, P.O. Box 1071, 3430 BB Nieuwegein, The Netherlands

e-mail: Kees.Maas@kwrwater.nl 


\section{Introduction}

After the derivation of the analytical solution for the steady, three-dimensional groundwater head in a system of $N$ coupled parallel, homogeneous aquifers (see [1], and for a numerical implementation [2,3]), interest has arisen to solve the transient case. A partial solution has been given by [4-6], where the authors consider vertical flow in aquitards and horizontal flow in aquifers. The first paper exhibits the analytical solution for a three-layer system. The two other ones give results for an $N$-layer system; the second one uses numerical inversion of the Laplace transform and the third one takes advantage of some special generalized Fourier transform (case $d_{i}=0$, to be explained in Sect. 3.3). Generalized Fourier transforms have been studied by, e.g., [7-11]. More information is also available from [12-16]. Many results for steady and some transient $N$-layer systems with no explicit dependence on the vertical variable have been published by Bruggeman [17, pp. 431-470].

We consider possible solution strategies and, contrary to existing approaches, we solve this problem without any restrictions. We treat aquifers and aquitards alike, i.e., both have horizontal and vertical permeabilities. More specifically, we discuss the influence of a well in such a system, but other hydrological features, such as natural groundwater flow, may be solved in a similar fashion. We shall use among others generalized Fourier transforms (case $d_{i} \neq 0$, to be explained in Sect.3.3). It turns out that an analytical solution in a strict sense does not seem to be possible, but we can specify the transformed solution either in terms of the variables $r$ and $z$, transform parameter $p$, and eigenvalues $\lambda_{m}^{2}(p)$ (27) or in terms of the variables $z$ and $t$, transform parameter $\alpha$, and eigenvalues $\lambda_{m}^{2}(\alpha)$ (31). These expressions need to be inverted numerically.

We discuss the notion of analytical solution in general, because, even if one cannot derive a fully analytical solution, partial results (after which one has to apply numerical techniques) are more informative than a purely numerical approach. The presentation of the analytical solution as an object which has to be inverted with respect to one or more of the independent variables $(t, r$, and $z$ ) numerically, gives more insight than to calculate the entire solution fully numerically by finite elements or finite diferences. Nowadays, it is possible to perform such inversions in a reasonable time, even if one has to recalculate eigenvalues many times due to dependencies on other transform parameters. See also [11, Chap. 3.2], [9] for a general account and references for more literature related to this topic.

\section{Layered system}

In this paper we study the transient groundwater head $\phi$ in a system of $N$ coupled parallel, homogeneous, horizontal aquifers. We denote $\phi_{i}[\mathrm{~L}]$ as the head in layer $i, i=1, \ldots, N$, numbered from the bottom-layer $(i=1)$ to the top-layer $(i=N)$. The $z$-axis is pointing upwards. So, the $i$-th aquifer is characterized by $L_{i-1} \leq z \leq L_{i}$, where $L_{0}=0$, and $L_{N}$ is the total thickness of the system. The thickness of an individual aquifer is thus $D_{i}=L_{i}-L_{i-1}$ [L]. We suppose that there is a well active at $r=0$, so we pose this groundwater head problem with radial symmetry in an infinite horizontal domain, but the general conclusions of this paper are also valid for cases with only natural groundwater flow caused by boundary conditions on a finite horizontal domain. We allow the horizontal permeability to be different from the vertical permeability $\left(K_{h, i}, K_{v, i}\left[\mathrm{LT}^{-1}\right]\right.$, respectively) for each aquifer. Also, the specific storativity $S_{s, i}\left[\mathrm{~L}^{-1}\right]$ is assumed to be different for each aquifer.

As initial condition we assume that the head $\phi_{i}(r, z, 0)$ equals $\phi_{i 0}(r, z)$, and at the bottom and top we assume general inhomogeneous boundary conditions of mixed type (also called Cauchy or Robin condition) such that the inflow is proportional to the head. The other two types of boundary conditions (Dirichlet, Neumann) can be derived from this general case. An important condition is the requirement of continuity of the head and the vertical flux at the common boundaries of the aquifers. The variable $Q_{i}\left[\mathrm{~L}^{3} \mathrm{~T}^{-1}\right]$ denotes the pumping rate of the well at $r=0$ with a partially penetrating filter with its center at $z=a_{i}$, and with length $l_{i}[\mathrm{~L}]$ in aquifer $i$. The variables $a_{i}$ and $l_{i}$ exist only if there is a well active in aquifer $i$, at $r=0$. One could treat cases where there is more than one partially penetrating filter at $r=0$ in an aquifer by the same method, through superposition.

All these conditions lead to the following system of partial differential equations with initial and boundary conditions, for $i=1, \ldots, N$ (where applicable): 
$S_{s, i} \frac{\partial \phi_{i}}{\partial t}=K_{h, i}\left(\frac{\partial^{2} \phi_{i}}{\partial r^{2}}+\frac{1}{r} \frac{\partial \phi_{i}}{\partial r}\right)+K_{v, i} \frac{\partial^{2} \phi_{i}}{\partial z^{2}}, \quad t>0, \quad r>0, \quad L_{i-1}<z<L_{i}$.

The initial condition is:

$\phi_{i}(r, z, 0)=\phi_{i 0}(r, z), \quad r>0, \quad L_{i-1}<z<L_{i}$.

The bottom and top boundary conditions are:

$\alpha_{0} \phi_{1}(r, 0, t)-\beta_{0} K_{v, 1} \frac{\partial \phi_{1}}{\partial z}(r, 0, t)=f_{b}(r, t), \quad t \geq 0, \quad r>0$,

$\alpha_{N} \phi_{N}\left(r, L_{N}, t\right)+\beta_{N} K_{v, N} \frac{\partial \phi_{N}}{\partial z}\left(r, L_{N}, t\right)=f_{t}(r, t), \quad t \geq 0, \quad r>0$,

where $\alpha_{0} \geq 0$, and $\beta_{0} \geq 0$, but not both $\alpha_{0}=\beta_{0}=0$,

and where $\alpha_{N} \geq 0$, and $\beta_{N} \geq 0$, but not both $\alpha_{N}=\beta_{N}=0$,

with $\left(\alpha_{0}, \alpha_{N}[-], \beta_{0}, \beta_{N}[\mathrm{~T}], f_{b}, f_{t}[\mathrm{~L}]\right)$. The interface conditions are:

$\phi_{i}\left(r, L_{i}, t\right)=\phi_{i+1}\left(r, L_{i}, t\right), \quad t \geq 0, \quad r>0, \quad i=1, \ldots, N-1$,

$K_{v, i} \frac{\partial \phi_{i}}{\partial z}\left(r, L_{i}, t\right)=K_{v, i+1} \frac{\partial \phi_{i+1}}{\partial z}\left(r, L_{i}, t\right), \quad t \geq 0, \quad r>0, \quad i=1, \ldots, N-1$.

The vertical boundary conditions are:

$$
\begin{array}{ll}
\lim _{r \rightarrow 0}\left(r \frac{\partial \phi_{i}}{\partial r}(r, z, t)\right)=\frac{Q_{i}}{2 \pi K_{h, i} l_{i}}, & t \geq 0, \quad a_{i}-l_{i} / 2 \leq z \leq a_{i}+l_{i} / 2, \\
& \text { i.e., } z \in A_{i}=\left[a_{i}-l_{i} / 2, a_{i}+l_{i} / 2\right], \\
\lim _{r \rightarrow 0}\left(r \frac{\partial \phi_{i}}{\partial r}(r, z, t)\right)=0, & t \geq 0, \quad L_{i-1} \leq z<a_{i}-l_{i} / 2 \text { and } a_{i}+l_{i} / 2<z \leq L_{i} .
\end{array}
$$

For the ease of presentation we assume from here on that $\phi_{i 0}(r, z), f_{b}(r, t), f_{t}(r, t)$ are all equal to zero. An arbitrary initial function or inhomogeneous boundary conditions will complicate the solution, but not essentially.

\section{Integral transforms}

To find the analytical solution for the system (1-3), (5), and (6) with three independent variables $(r, z, t)$ one usually applies integral transforms to get rid off the differentials; see for a general account [18, Chaps. 2, 3, and 5]. For the equations of (1) one has the possibility to apply the Laplace transform with respect to $t$, the Hankel transform with respect to $r$, and a generalized Fourier transform in the $z$-direction which takes care of the interface conditions. More information with respect to these three transforms is given in the following.

\subsection{Laplace transform}

$\mathrm{L}[\phi](p)=\bar{\phi}(r, z, p)=\int_{0}^{\infty} \phi(r, z, t) \exp (-p t) \mathrm{d} t$,

with the main property for our problem

$$
\int_{0}^{\infty} \frac{\partial \phi(r, z, t)}{\partial t} \exp (-p t) \mathrm{d} t=p \bar{\phi}(r, z, p)-\phi(r, z, 0) .
$$

The theoretical general form for the inverse of the Laplace transform reads $\left(\bar{\phi}(r, z, p)\right.$ exists for $\left.\mathfrak{R e}(p)>p_{0}\right)$ :

$\mathrm{L}^{-1}[\bar{\phi}](r, z, t)=\phi(r, z, t)=\frac{1}{2 \pi \mathrm{i}} \int_{L} \bar{\phi}(r, z, p) \exp (p t) \mathrm{d} p$,

where $L=\left\{s \mid s=\left(p_{1}, \mathrm{i} q\right), p_{1}>p_{0}, p_{1}\right.$ fixed, $\left.-\infty<q<\infty\right\}$, the Bromwich contour. 
3.2 Hankel transform

$\mathrm{H}[\phi](\alpha)=\widehat{\phi}(\alpha, z, t)=\int_{0}^{\infty} \phi(r, z, t) r \mathrm{~J}_{0}(\alpha r) \mathrm{d} r$,

with the main property for our problem:

$$
\int_{0}^{\infty}\left(\frac{\partial^{2} \phi(r, z, t)}{\partial r^{2}}+\frac{1}{r} \frac{\partial \phi(r, z, t)}{\partial r}\right) r \mathrm{~J}_{0}(\alpha r) \mathrm{d} r=-\alpha^{2} \widehat{\phi}(\alpha, z, t)-\lim _{r \rightarrow 0}\left(r \frac{\partial \phi(r, z, t)}{\partial r}\right) .
$$

The inverse transform for the Hankel transform reads:

$\mathrm{H}^{-1}[\widehat{\phi}](r, z, t)=\phi(r, z, t)=\int_{0}^{\infty} \widehat{\phi}(\alpha, z, t) \alpha \mathrm{J}_{0}(\alpha r) \mathrm{d} \alpha$.

\subsection{Generalized Fourier transform}

Here we denote by $\phi(r, z, t)=\left\{\phi_{i}(r, z, t)\right\}_{i=1}^{i=N}$, i.e., the collection of the functions $\phi_{i}(r, z, t)$, where for given index $i: L_{i-1}<z<L_{i}$. The generalized Fourier transform reads for some given constants $w_{i}\left(w_{i}>0\right)$ :

$\mathrm{Z}[\phi](m)=\phi^{m}(r, t)=\sum_{i=1}^{N} \int_{L_{i-1}}^{L_{i}} \phi_{i}(r, z, t) w_{i} \psi_{i}^{m}(z) \mathrm{d} z, \quad m=1,2, \ldots$,

where the function $\psi^{m}(z)=\left\{\psi_{i}^{m}(z)\right\}_{i=1}^{i=N}, m=1,2, \ldots$ is the $m$ th eigenfunction of the generalized eigenvalue problem defined by (13-15), with $\mu_{m}=\lambda_{m}^{2}$, the $m$ th eigenvalue. So, for each subinterval

$K_{v, i} \frac{\mathrm{d}^{2} \psi_{i}^{m}(z)}{\mathrm{d} z^{2}}=-\lambda_{m}^{2} w_{i} \psi_{i}^{m}(z)+d_{i} \psi_{i}^{m}(z), \quad L_{i-1}<z<L_{i}, \quad i=1, \ldots, N$,

where the positive constants $w_{i}$ and $d_{i}$ are to be chosen appropriately, depending on the problem. The bottom and top boundary conditions are

$\alpha_{0} \psi_{1}(0)-\beta_{0} K_{v, 1} \frac{\mathrm{d} \psi_{1}^{m}}{\mathrm{~d} z}(0)=0, \alpha_{N} \psi_{N}\left(L_{N}\right)+\beta_{N} K_{v, N} \frac{\mathrm{d} \psi_{N}^{m}}{\mathrm{~d} z}\left(L_{N}\right)=0$,

with the requirement (4), and the interface conditions

$\begin{array}{ll}\psi_{i}^{m}\left(L_{i}\right)=\psi_{i+1}^{m}\left(L_{i}\right), & i=1, \ldots, N-1, \\ K_{v, i} \frac{\mathrm{d} \psi_{i}^{m}}{\mathrm{~d} z}\left(L_{i}\right)=K_{v, i+1} \frac{\mathrm{d} \psi_{i+1}^{m}}{\mathrm{~d} z}\left(L_{i}\right), & i=1, \ldots, N-1 .\end{array}$

The main property of this transform reads $\left(\mathrm{Z}[\phi](m)=\phi^{m}(r, t)\right.$, i.e., the transformed function $\left.\phi\right)$ :

$$
\begin{aligned}
& \sum_{i=1}^{N} \int_{L_{i-1}}^{L_{i}} w_{i}^{-1}\left(K_{v, i} \frac{\partial^{2} \phi_{i}}{\partial z^{2}}(r, z, t)-d_{i} \phi_{i}(r, z, t)\right) w_{i} \psi_{i}^{m}(z) \mathrm{d} z=-\lambda_{m}^{2} \mathrm{Z}[\phi](m) \\
& \quad-K_{v, 1}\left(\psi_{1}^{m}(0) \frac{\partial \phi_{1}}{\partial z}(r, 0, t)-\frac{\mathrm{d} \psi_{1}^{m}}{\mathrm{~d} z}(0) \phi_{1}(r, 0, t)\right) \\
& \quad+K_{v, N}\left(\psi_{N}^{m}\left(L_{N}\right) \frac{\partial \phi_{N}}{\partial z}\left(r, L_{N}, t\right)-\frac{\mathrm{d} \psi_{N}^{m}}{\mathrm{~d} z}\left(L_{N}\right) \phi_{N}\left(r, L_{N}, t\right)\right) .
\end{aligned}
$$


So, for this choice of $\psi^{m}(z)$ the second-order differentials of $\phi$ transform to the transform of $\phi$. Given the boundary values at the top and bottom in (3), Eq. 16 reduces to

$$
\begin{aligned}
& \sum_{i=1}^{N} \int_{L_{i-1}}^{L_{i}} w_{i}^{-1}\left(K_{v, i} \frac{\partial^{2} \phi_{i}}{\partial z^{2}}(r, z, t)-d_{i} \phi_{i}(r, z, t)\right) w_{i} \psi_{i}^{m}(z) \mathrm{d} z \\
& \quad=-\lambda_{m}^{2} \mathrm{Z}[\phi](m)+\frac{1}{\beta_{0}} \psi_{1}^{m}(0) f_{b}(r, t)+\frac{1}{\beta_{N}} \psi_{N}^{m}\left(L_{N}\right) f_{t}(r, t) .
\end{aligned}
$$

If $\beta_{0}=0$, the term in (17) with $\beta_{0}$ has to be changed into $\frac{K_{v, 1}}{\alpha_{0}} \frac{\mathrm{d} \psi_{1}^{m}}{\mathrm{~d} z}(0) f_{b}(r, t)$, and if $\beta_{N}=0$, the term with $\beta_{N}$ into $-\frac{K_{v, N}}{\alpha_{N}} \frac{\mathrm{d} \psi_{N}^{m}}{\mathrm{~d} z}\left(L_{N}\right) f_{t}(r, t)$. See [2] for details with respect to the actual construction of the eigenfunctions for the restricted case of $d_{i}=0$.

With the requirement (4) it can be shown that indeed $\lambda_{m}^{2}>0$ as follows. Multiply (13) by $\psi_{i}^{m}(z)$, integrate over $\left(L_{i-1}, L_{i}\right)$, and sum over $i=1, \ldots, N$. Then one finds, for $\beta_{0}>0$, and $\beta_{N}>0$

$$
\begin{aligned}
\lambda_{m}^{2} \sum_{i=1}^{N} \int_{L_{i-1}}^{L_{i}} w_{i}\left(\psi_{i}^{m}(z)\right)^{2} \mathrm{~d} z= & \sum_{i=1}^{N} \int_{L_{i-1}}^{L_{i}}\left\{-K_{v, i} \frac{\mathrm{d}^{2} \psi_{i}^{m}(z)}{\mathrm{d} z^{2}}+d_{i} \psi_{i}^{m}(z)\right\} \psi_{i}^{m}(z) \mathrm{d} z \\
= & \sum_{i=1}^{N} \int_{L_{i-1}}^{L_{i}}\left\{K_{v, i}\left(\frac{\mathrm{d} \psi_{i}^{m}(z)}{\mathrm{d} z}\right)^{2}+d_{i}\left(\psi_{i}^{m}(z)\right)^{2}\right\} \mathrm{d} z-\left.\sum_{i=1}^{N} K_{v, i} \frac{\mathrm{d} \psi_{i}^{m}(z)}{\mathrm{d} z} \psi_{i}^{m}(z)\right|_{L_{i-1}} ^{L_{i}} \\
= & \sum_{i=1}^{N} \int_{L_{i-1}}^{L_{i}}\left\{K_{v, i}\left(\frac{\mathrm{d} \psi_{i}^{m}(z)}{\mathrm{d} z}\right)^{2}+d_{i}\left(\psi_{i}^{m}(z)\right)^{2}\right\} \mathrm{d} z-K_{v, N} \frac{\mathrm{d} \psi_{N}^{m}\left(L_{N}\right)}{\mathrm{d} z} \psi_{N}^{m}\left(L_{N}\right) \\
& +K_{v, 1} \frac{\mathrm{d} \psi_{1}^{m}(0)}{\mathrm{d} z} \psi_{1}^{m}(0) \\
= & \sum_{i=1}^{N} \int_{L_{i-1}}^{L_{i}}\left\{K_{v, i}\left(\frac{\mathrm{d} \psi_{i}^{m}(z)}{\mathrm{d} z}\right)^{2}+d_{i}\left(\psi_{i}^{m}(z)\right)^{2}\right\} \mathrm{d} z+\frac{\alpha_{N}}{\beta_{N}}\left(\psi_{N}^{m}\left(L_{N}\right)\right)^{2} \\
& +\frac{\alpha_{0}}{\beta_{0}}\left(\psi_{1}^{m}(0)\right)^{2}>0,
\end{aligned}
$$

due to the requirement (4) and the fact that $K_{v, i}, w_{i}$ and $d_{i}$ are all positive. So it follows that also $\mu_{m}=\lambda_{m}^{2}>0$. If $\beta_{0}=0$, the term $K_{v, 1} \frac{\mathrm{d} \psi_{1}^{m}(0)}{\mathrm{d} z} \psi_{1}^{m}(0)=0$, and if $\beta_{N}=0$, the term $-K_{v, N} \frac{\mathrm{d} \psi_{N}^{m}\left(L_{N}\right)}{\mathrm{d} z} \psi_{N}^{m}\left(L_{N}\right)=0$, so the same conclusion holds.

The construction of the eigenvalues and the eigenfunctions runs as follows (see also [11, pp. 115-120]). On each $z$-interval the function $\psi_{i}^{m}(z), L_{i-1}<z<L_{i}$, consists of sine functions

$\psi_{i}^{m}(z)=\frac{F_{i-1}^{m} \sin \left(R_{i}^{m}\left(L_{i}-z\right)\right)+F_{i}^{m} \sin \left(R_{i}^{m}\left(z-L_{i-1}\right)\right)}{\sin \left(R_{i}^{m} D_{i}\right)}, \quad R_{i}^{m}=\sqrt{\left(\lambda_{m}^{2} w_{i}-d_{i}\right) / K_{v, i}}$.

So, $\psi_{i}^{m}\left(L_{i-1}\right)=F_{i-1}^{m}$ and $\psi_{i}^{m}\left(L_{i}\right)=F_{i}^{m}$, and by the choice of this representation the first requirement in (15) is fullfilled immediately. To find the values $\lambda_{m}$ and the corresponding $\left\{F_{i}^{m}\right\}_{i=0}^{N}$ we construct a $(N+1 \times N+1)$-matrix using the conditions (14) and the second requirement in (15). That will lead to the matrix $G$ with equation 


$$
\left[\begin{array}{cccccccc}
a_{1} & -b_{1} & 0 & 0 & \cdots & 0 & 0 & 0 \\
-b_{1} & a_{2} & -b_{2} & 0 & \cdots & 0 & 0 & 0 \\
0 & -b_{2} & a_{3} & -b_{3} & \cdots & 0 & 0 & 0 \\
0 & 0 & -b_{3} & a_{4} & \cdots & 0 & 0 & 0 \\
\cdots & \cdots & \cdots & \cdots & \cdots & \cdots & \cdots & \cdots \\
0 & 0 & 0 & 0 & \cdots & a_{N-1} & -b_{N-1} & 0 \\
0 & 0 & 0 & 0 & \cdots & -b_{N-1} & a_{N} & -b_{N} \\
0 & 0 & 0 & 0 & \cdots & 0 & -b_{N} & a_{N+1}
\end{array}\right]\left[\begin{array}{l}
F_{0}^{m} \\
F_{1}^{m} \\
F_{2}^{m} \\
F_{3}^{m} \\
\cdots \\
F_{N-2}^{m} \\
F_{N-1}^{m} \\
F_{N}^{m}
\end{array}\right]=\left[\begin{array}{l}
0 \\
0 \\
0 \\
0 \\
\cdots \\
0 \\
0 \\
0
\end{array}\right]
$$

where the coefficients are given by

$$
\begin{aligned}
a_{i} & =A_{i-1}+A_{i}, \quad i=1,2, \ldots, N+1, \\
b_{i} & =B_{i}=\frac{K_{v, i} R_{i}^{m}}{\sin \left(R_{i}^{m} D_{i}\right)}, \quad i=1,2, \ldots, N, \\
A_{i} & =B_{i} \cos \left(R_{i}^{m} D_{i}\right), \quad i=1,2, \ldots, N, \\
A_{0} & =\frac{\alpha_{0}}{\beta_{0}}, \quad A_{N+1}=\frac{\alpha_{N}}{\beta_{N}} .
\end{aligned}
$$

For the cases where $\beta_{0}=0$, or $\beta_{N}=0$, or $\beta_{0}=\beta_{N}=0$, one can simply remove the corresponding $\operatorname{row}(\mathrm{s})$ and column(s) from the matrix $G$. The eigenvalues $\lambda_{m}^{2}$ follow from the requirement that $\operatorname{det}(G)=0$. This will be a transcedental equation with an infinite number of values $\lambda_{m}^{2}, m=1,2, \ldots$ In cases where $\lambda_{m}^{2} w_{i}-d_{i}<0$, one simply changes in (18) and (20) the coefficient $R_{i}^{m}$ into $R_{i}^{m}=\sqrt{\left|\lambda_{m}^{2} w_{i}-d_{i}\right| / K_{v, i}}$ and the functions sin and cos into sinh and cosh, respectively. For the case $\lambda_{m}^{2} w_{i}-d_{i}=0$, the eigenfunction becomes $\psi_{i}^{m}(z)=$ $\left(F_{i-1}^{m}\left(L_{i}-z\right)+F_{i}^{m}\left(z-L_{i-1}\right)\right) / D_{i}$ and $A_{i}=B_{i}=K_{v, i} / D_{i}$, see [19]. From the general Sturm-Liouville theory we know that the smallest eigenvalue gives rise to an eigenfunction with no zeros in the interval $\left(0, L_{N}\right)$, see [20, Chap. 4]. The Sign-Count method [21, 19] is useful for the actual calculation of the eigenvalues.

For the generalized Fourier transform the inverse reads:

$\mathrm{Z}^{-1}\left[\phi^{m}\right](r, z, t)=\phi(r, z, t)=\left\{\phi_{i}(r, z, t)\right\}_{i=1}^{i=N}$,

where

$\phi_{i}(r, z, t)=\sum_{m=1}^{\infty} \phi^{m}(r, t) \psi_{i}^{m}(z) / N_{m}, \quad$ with $N_{m}=\sum_{i=1}^{N} \int_{L_{i-1}}^{L_{i}} w_{i}\left\{\psi_{i}^{m}(z)\right\}^{2} \mathrm{~d} z$.

The terms in the summation of $N_{m}$ can be written in terms of the coefficients $A_{i}$ and $B_{i}$ as

$$
\int_{L_{i-1}}^{L_{i}}\left\{\psi_{i}^{m}(z)\right\}^{2} \mathrm{~d} z=\frac{1}{2\left(\lambda_{m}^{2} w_{i}-d_{i}\right)}\left\{\left(\left(F_{i-1}^{m}\right)^{2}+\left(F_{i}^{m}\right)^{2}\right)\left(\frac{B_{i}^{2} D_{i}}{K_{v, i}}-A_{i}\right)+2 B_{i} F_{i-1}^{m} F_{i}^{m}\left(1-\frac{A_{i} D_{i}}{K_{v, i}}\right)\right\},
$$

which is valid both for the cases where $\lambda_{m}^{2} w_{i}-d_{i}>0$ and $\lambda_{m}^{2} w_{i}-d_{i}<0$. If $\lambda_{m}^{2} w_{i}-d_{i}=0$, there holds

$\int_{L_{i-1}}^{L_{i}}\left\{\psi_{i}^{m}(z)\right\}^{2} \mathrm{~d} z=\frac{D_{i}}{3}\left(\left(F_{i-1}^{m}\right)^{2}+\left(F_{i}^{m}\right)^{2}+F_{i-1}^{m} F_{i}^{m}\right)$.

\subsection{Solution technique}

After one has applied two integral transforms, there remains an ordinary differential equation in one of the variables $(r, z, t)$. Solving this ordinary differential equation in terms of either $t$ or $r$ is not so difficult; the resulting ordinary differential equation in $z$ is more complicated. Having found the solution of the ordinary differential equation, 
one has to apply the inverse transformations of the respective integral transforms. For the Laplace transforms the inverse transform can be given for quite a number of functions. In cases that one has to deal with functions which cannot be found analytically, it is important that the inverse transforms can be found by an inversion technique using only real values for $p$ (such as the Gaver-Stehfest method [22]), because the analysis below shows that complex $p$-values complicate the other transforms (some fast numerical methods for the Laplace inversion (such as [23]) require complex values of the transform parameter $p$ ).

Below, we shall specify the resulting ordinary differential equation for the three different cases (in $r, t, z$, respectively), but first, we discuss the notion of an analytical solution.

\section{Classification of analytical solutions}

The aim of constructing analytical solutions is to learn as much as possible about the nature of the model under investigation, since they allow the unknown variable to be presented in terms of the independent variables by means of more or less known functions (exponentials (exp, sin, cos, sinh, cosh), logarithms) and functions like Bessel-functions, erfc-functions or functions which can be calculated by a mathematical algorithm. The increase in power of the computing devices has made it possible to compute such functions whereas before one had to rely on tables and interpolation. Knowledge of the analytical expression teaches us a lot about limit situations, asymptotic behaviour and other special cases. It also helps to determine the sensitivity of the solution with respect to the parameters. Moreover, one can easily determine the derivatives with respect to the spatial variables which is helpful for particle tracking or gradient-based parameter estimation for cases where the groundwater head is the dependent variable. And finally, knowledge of the analytical solution of a problem serves as an independent check on a strictly numerical procedure to calculate the solution. The utility of analytical solutions for these purposes depends on their degree of explicitness.

Therefore, we propose a classification of analytical solutions according to their degree of explicitness, starting with the most explicit class. Inevitably, the class boundaries will be arbitrary to some extent.

Class 1 . In this case the solution can be expressed in terms of elementary functions and can be calculated in a straightforward manner. Included in this class are infinite summations and integrals over elementary functions.

Class 2. This class comes close to Class 1, but differs by the fact that one has to perform some explicit calculation to find some unknown constants before one can apply the procedure under Class 1 . For example, some boundary-value problems can be expressed as infinite sums that include numbers $\lambda_{n}$, that have to be calculated as roots of some transcendental equation (e.g. $\lambda_{n} \tan \lambda_{n}=C$, in which $C$ encompasses an expression with parameters of the problem).

Class 3. This class resembles Classes 1 and 2, but differs in the sense that certain unknown constants need to be solved for (e.g. zeros of the determinant of a matrix) in such a way that one can hardly infer anything about the nature of these constants based on the parameters in the problem (e.g. asymptotic behaviour). The solution of groundwater problems by means of the analytic-element method [24] belong to this class.

Class 4. Under this class we consider all analytical solutions of an integral transform for which there is no direct inverse available and one has to apply some kind of numerical transform. Under this class we reckon all explicitly expressible Laplace transforms; this means that there are no constants involved which have to be calculated numerically. Sometimes, the inverse Laplace transform is explicitly available, so these functions should belong to Class 1 or 2, but their expressions become cumbersome and numerical inversion is more straightforward and even more stable in a numerical sense.

Class 5. The same as Class 4, but now we have to deal with unknown constants in the integral transform specified by a transcedental equation (as under Class 3). The difference between Class 4 and Class 5 is motivated by the fact that for expressions that fall under Class 4 it is often possible to derive the asymptotic behaviour (e.g. for Laplace transforms for small $t$ and large $t$ ), which is impossible for expressions in this class. 
Class 6. The same as Class 5, but now the constants which have to be solved for in the integral-transform depend on the integral transform parameter in an implicit way. Under this class we reckon those analytical expressions in which two integral transforms are involved and for which the first inverse transform depends in an implicit way on the second integral transform parameter (or vice versa). Examples of solutions for this Class 6 can be found in [25], where transient flow has been treated using the analytic-element method and in [5], where flow is considered in layered and fissured aquifer systems.

Class 7. The same as Class 6, but for this class the first integral transform depends on two other integral-transform parameters. Examples for this class are given in [26] and [27].

Sometimes, solutions belonging to Classes 5, 6 and 7 are called semi-analytical.

\section{Integral transforms}

We analyze the three main possibilities, for solving problem (1-3), (5), and (6), which results in an ordinary differential equation in $r, t$ or $z$. We remark that the order in which one applies the transformations is not important. In the following we shall assume that the functions $\phi_{i 0}(r, z), f_{b}(r, t)$ and $f_{t}(r, t)$, which represent the initial condition, the bottom boundary and the top boundary, respectively, equal zero, but we note that the construction of the semi-analytical solutions is still possible without these restrictions. Under these assumptions the presence of the well ensures that $\phi_{i}(r, z, t) \not \equiv 0$.

\subsection{Integral transform in terms of $t$ and $z$}

After application of the Laplace transform, we find:

$S_{s, i} p \bar{\phi}_{i}=K_{h, i}\left(\frac{\partial^{2} \bar{\phi}_{i}}{\partial r^{2}}+\frac{1}{r} \frac{\partial \bar{\phi}_{i}}{\partial r}\right)+K_{v, i} \frac{\partial^{2} \bar{\phi}_{i}}{\partial z^{2}}, \quad r>0, \quad L_{i-1}<z<L_{i}$.

We proceed by applying the generalized Fourier transform (12) with the following choices: $w_{i}=K_{h, i}$ and $d_{i}=$ $S_{s, i} p$. Multiplication of (24) by $\psi_{i}^{m}(z)$, and integration over $\left(L_{i-1}, L_{i}\right)$, and summation over $i=1, \ldots, N$ gives (using (17)):

$0=\left(\frac{\mathrm{d}^{2} \mathrm{Z}[\bar{\phi}](m)}{\mathrm{d} r^{2}}+\frac{1}{r} \frac{\mathrm{dZ}[\bar{\phi}](m)}{\mathrm{d} r}\right)-\lambda_{m}^{2} \mathrm{Z}[\bar{\phi}](m), \quad r>0, \quad m=1, \ldots$,

$\lim _{r \rightarrow 0}\left(r \frac{\mathrm{dZ}[\bar{\phi}](m)}{\mathrm{d} r}\right)=\frac{1}{p} \widetilde{Q}(m), \quad$ with $\widetilde{Q}(m)=\sum_{i=1}^{N} \int_{L_{i-1}}^{L_{i}} \frac{Q_{i}}{2 \pi l_{i}} \mathrm{I}_{A_{i}}(z) \psi_{i}^{m}(z) \mathrm{d} z$,

and where

$$
\mathrm{I}_{A_{i}}(z)=1, \quad a_{i}-l_{i} / 2 \leq z \leq a_{i}+l_{i} / 2
$$

$$
\mathrm{I}_{A_{i}}(z)=0, \quad L_{i-1} \leq z<a_{i}-l_{i} / 2 \text { and } a_{i}+l_{i} / 2<z \leq L_{i} .
$$

Equation 25 together with the transform of boundary condition (6) can be solved in terms of the Modified Bessel function of order zero, $\mathrm{K}_{0}$, as

$\mathrm{Z}[\bar{\phi}](m)=-\frac{1}{p} \widetilde{Q}(m) \mathrm{K}_{0}\left(\lambda_{m} r\right)$.

Notice that in this case $\lambda_{m}=\lambda_{m}(p)$, and so $N_{m}=N_{m}(p)$. The full solution has the representation

$\phi(r, z, t)=\left\{\phi_{i}(r, z, t)\right\}_{i=1}^{i=N}, \quad$ with $\phi_{i}(r, z, t)=-\mathrm{L}^{-1}\left[\frac{1}{p} \sum_{m=1}^{\infty} \widetilde{Q}(m) \mathrm{K}_{0}\left(\lambda_{m} r\right) \psi_{i}^{m}(z) / N_{m}\right]$.

Although the full solution looks quite simple, to evaluate this function a lot of work has to be done to calculate the eigenvalues $\lambda_{m}^{2}$ for every value of the transform parameter $p$ that is required during the Laplace-transform inversion. 
Clearly, this solution belongs to Class 6, described in Sect. 4. This representation is particularly useful for particle tracking after explicit differentiation of (27) with respect to $r$ and $z$.

As a side remark we mention the fact that in the quite restrictive case $K_{h, i} / S_{s, i}=\rho$, independent of $i$, we can apply the generalized Fourier transform with $d_{i}=0$, moving the solution to Class 3; see the Appendix A for details.

\subsection{Integral transform in terms of $r$ and $z$}

After an application of the Hankel transform, we find (for $i=1, \ldots, N$ )

$$
\begin{array}{ll}
S_{s, i} \frac{\partial \widehat{\phi}_{i}}{\partial t}=-K_{h, i} \alpha^{2} \widehat{\phi}_{i}-\frac{Q_{i}}{2 \pi l_{i}}+K_{v, i} \frac{\partial^{2} \widehat{\phi}_{i}}{\partial z^{2}}, & t>0, \quad a_{i}-l_{i} / 2 \leq z \leq a_{i}+l_{i} / 2, \\
S_{s, i} \frac{\partial \widehat{\phi}_{i}}{\partial t}=-K_{h, i} \alpha^{2} \widehat{\phi}_{i}+K_{v, i} \frac{\partial^{2} \widehat{\phi}_{i}}{\partial z^{2}}, & t>0, \quad L_{i-1} \leq z<a_{i}-l_{i} / 2 \text { and } a_{i}+l_{i} / 2<z \leq L_{i},
\end{array}
$$

where we denote by $\widehat{\phi}_{i}$ the function $\widehat{\phi}_{i}(\alpha, z, t)$. Next, we apply the generalized Fourier transform (with $w_{i}=S_{S, i}$ and $\left.d_{i}=K_{h, i} \alpha^{2}\right)$ :

$$
\frac{\mathrm{dZ}[\widehat{\phi}](m)}{\mathrm{d} t}=-\widetilde{Q}(m)-\lambda_{m}^{2} \mathrm{Z}[\widehat{\phi}](m), \quad t>0, \quad m=1, \ldots,
$$

where $\widetilde{Q}(m)$ has been defined in $(25)$,

$\mathrm{Z}[\widehat{\phi}](m)=0, \quad t=0$.

This equation can easily be solved as

$\mathrm{Z}[\widehat{\phi}](m)=-\widetilde{Q}(m)\left(\frac{1-\exp \left(-\lambda_{m}^{2} t\right)}{\lambda_{m}^{2}}\right)$,

Notice that in this case $\lambda_{m}=\lambda_{m}\left(\alpha^{2}\right)$, and so $N_{m}=N_{m}\left(\alpha^{2}\right)$. The full solution has the representation

$\phi(r, z, t)=\left\{\phi_{i}(r, z, t)\right\}_{i=1}^{i=N}$,

with $\phi_{i}(r, z, t)=-\int_{0}^{\infty}\left\{\sum_{m=1}^{\infty} \widetilde{Q}(m)\left(\frac{1-\exp \left(-\lambda_{m}^{2} t\right)}{\lambda_{m}^{2}}\right) \psi_{i}^{m}(z) / N_{m}\right\} \alpha \mathrm{J}_{0}(\alpha r) \mathrm{d} \alpha$.

Once more, to evaluate this function a lot of work has to be done to calculate the eigenvalues $\lambda_{m}^{2}$ for every value of the transform parameter $\alpha$ required during the integration over $\alpha$. Clearly, this presentation of the solution belongs also to Class 6, described in Sect. 4.

Again, as a side remark we mention that, if $K_{h, i} / S_{s, i}=\rho$, the solution becomes simpler, moving the solution to Class 3; see the Appendix B.

\subsection{Integral transform in terms of $t$ and $r$}

Application of the Laplace transform (7) to (1) gives (24), where we denote by $\bar{\phi}_{i}$ the function $\bar{\phi}_{i}(r, z, p)$. Application of the Hankel transform (9) gives

$$
\begin{array}{ll}
S_{s, i} p \widehat{\bar{\phi}}_{i, b}=-K_{h, i} \alpha^{2} \widehat{\bar{\phi}}_{i, b}+K_{v, i} \frac{\mathrm{d}^{2} \widehat{\bar{\phi}}_{i, b}}{\mathrm{~d} z^{2}}, & L_{i-1} \leq z<a_{i}-l_{i} / 2, \\
S_{s, i} p \widehat{\bar{\phi}}_{i, w}=-K_{h, i} \alpha^{2} \widehat{\bar{\phi}}_{i, w}-\frac{1}{p} \frac{Q_{i}}{2 \pi l_{i}}+K_{v, i} \frac{\mathrm{d}^{2} \widehat{\bar{\phi}}_{i, w}}{\mathrm{~d} z^{2}}, & a_{i}-l_{i} / 2 \leq z \leq a_{i}+l_{i} / 2, \\
S_{s, i} p \widehat{\bar{\phi}}_{i, t}=-K_{h, i} \alpha^{2} \widehat{\bar{\phi}}_{i, t}+K_{v, i} \frac{\mathrm{d}^{2} \overline{\bar{\phi}}_{i, t}}{\mathrm{~d} z^{2}}, & a_{i}+l_{i} / 2<z \leq L_{i},
\end{array}
$$


where we denote by $\widehat{\bar{\phi}}_{i, s}$ the function $\widehat{\bar{\phi}}_{i, s}(\alpha, z, p), s=b, w, t$ (for the bottom-, well-, top-sublayer, respectively). In (32) the conditions (2) and (6) have been incorporated. The coupled ordinary differential equations (32) have to be solved subject to the transformed versions of boundary conditions (3) and (5), namely

$\alpha_{0} \widehat{\bar{\phi}}_{1, b}(\alpha, 0, p)-\beta_{0} K_{v, 1} \frac{\mathrm{d} \widehat{\bar{\phi}}_{1, b}}{\mathrm{~d} z}(\alpha, 0, p)=0$,
$\alpha_{N} \widehat{\bar{\phi}}_{N, t}(\alpha, 0, p)+\beta_{N} K_{v, N} \frac{\mathrm{d} \widehat{\bar{\phi}}_{N, t}}{\mathrm{~d} z}\left(\alpha, L_{N}, p\right)=0$,

and

$\widehat{\bar{\phi}}_{i, t}\left(\alpha, L_{i}, p\right)=\widehat{\bar{\phi}}_{i+1, b}\left(\alpha, L_{i}, p\right), \quad i=1, \ldots, N-1$,

$K_{v, i} \frac{\mathrm{d} \widehat{\bar{\phi}}_{i, t}}{\mathrm{~d} z}\left(\alpha, L_{i}, p\right)=K_{v, i+1} \frac{\mathrm{d} \widehat{\bar{\phi}}_{i+1, b}}{\mathrm{~d} z}\left(\alpha, L_{i}, p\right), \quad i=1, \ldots, N-1$,

and with the continuity conditions at the levels of the partially penetrating filters

$\widehat{\bar{\phi}}_{i, b}\left(\alpha, a_{i}-l_{i} / 2, p\right)=\widehat{\bar{\phi}}_{i, w}\left(\alpha, a_{i}-l_{i} / 2, p\right), \quad i=1, \ldots, N$,
$\frac{\mathrm{d} \widehat{\bar{\phi}}_{i, t}}{\mathrm{~d} z}\left(\alpha, a_{i}-l_{i} / 2, p\right)=\frac{\mathrm{d} \widehat{\bar{\phi}}_{i, w}}{\mathrm{~d} z}\left(\alpha, a_{i}-l_{i} / 2, p\right), \quad i=1, \ldots, N$,

and

$\widehat{\bar{\phi}}_{i, w}\left(\alpha, a_{i}+l_{i} / 2, p\right)=\widehat{\bar{\phi}}_{i, t}\left(\alpha, a_{i}+l_{i} / 2, p\right), \quad i=1, \ldots, N$,

$\frac{\mathrm{d} \widehat{\bar{\phi}}_{i, w}}{\mathrm{~d} z}\left(\alpha, a_{i}+l_{i} / 2, p\right)=\frac{\mathrm{d} \widehat{\bar{\phi}}_{i, t}}{\mathrm{~d} z}\left(\alpha, a_{i}+l_{i} / 2, p\right), \quad i=1, \ldots, N$.

Next, we apply the generalized Fourier transform to $\widehat{\bar{\phi}}_{i}$ choosing $w_{i}=S_{s, i} p+K_{h, i} \alpha^{2}, d_{i}=0$. Two other choices apply as well, namely $\left\{w_{i}=S_{s, i} p, d_{i}=K_{h, i} \alpha^{2}\right\}$ and $\left\{w_{i}=K_{h, i} \alpha^{2}, d_{i}=S_{s, i} p\right\}$. Both lead to the same equations as below, but the corresponding eigenvalues and eigenfunctions are different, because the defining ordinary differential equation for the eigenfunctions is different. Consequently, also $\widetilde{Q}(m)$ is different for the three choices.

$\mathrm{Z}[\widehat{\bar{\phi}}](m)=-\frac{1}{p} \widetilde{Q}(m)-\lambda_{m}^{2} \mathrm{Z}[\widehat{\widehat{\phi}}](m), \quad t>0, \quad m=1, \ldots$,

from which one can infer that

$\mathrm{Z}[\widehat{\bar{\phi}}](m)=-\frac{\widetilde{Q}(m)}{p\left(1+\lambda_{m}^{2}\right)}$.

Finally, the solution of the full problem can be written as

$\phi_{i}(r, z, t)=-\mathrm{L}^{-1}\left[\int_{0}^{\infty}\left\{\sum_{m=1}^{\infty} \frac{\widetilde{Q}(m)}{p\left(1+\lambda_{m}^{2}\right)} \psi_{i}^{m}(z) / N_{m}\right\} \alpha \mathrm{J}_{0}(\alpha r) \mathrm{d} \alpha\right], \quad i=1, \ldots, N$.

(in this case $\lambda_{m}=\lambda_{m}\left(p, \alpha^{2}\right)$, and so $N_{m}=N_{m}\left(p, \alpha^{2}\right)$ ). Now one has to calculate the eigenvalues $\lambda_{m}^{2}$ for every value of the transform parameters $p$ and $\alpha^{2}$. The analytical solution found this way belongs to Class 7 , as described in Sect. 4.

If one assumes the restrictive hypothesis that $K_{h, i} / S_{s, i}=\rho$, independent of $i$, one takes $w_{i}=S_{s, i}, d_{i}=0$, the solution becomes simpler, moving the solution to Class 3; see the Appendix C for details.

Another way to solve the resulting coupled ordinary differential equations (32) with (33-37) can be performed in a straightforward manner as was done in [26] for a comparable case with a natural groundwater flow in the horizontal $x$-direction and a prescribed flux at $x=0$ over the full height of the corresponding aquifer instead of 
the case here with a partially penetrating filter. The solution for the case $\alpha_{0}=\alpha_{N}=0$ in (33) and (34) can be represented by (for $i=1, \ldots, N$, and with the shorthand notation $R_{i}=\sqrt{\left(S_{s, i} p+K_{h, i} \alpha^{2}\right) / K_{v, i}}$ ),

$$
\begin{aligned}
\widehat{\bar{\phi}}_{i, b}(\alpha, z, p)= & \frac{F_{i, b} \cosh \left(R_{i}\left(z-\left(a_{i}-l_{i} / 2\right)\right)\right)-F_{i, w} \cosh \left(R_{i}\left(z-L_{i-1}\right)\right)}{K_{v, i} R_{i} \sinh \left(R_{i}\left(\left(a_{i}-l_{i} / 2\right)-L_{i-1}\right)\right)}, & L_{i-1} \leq z<a_{i}-l_{i} / 2, \\
\widehat{\bar{\phi}}_{i, w}(\alpha, z, p)= & \frac{F_{i, w} \cosh \left(R_{i}\left(z-\left(a_{i}+l_{i} / 2\right)\right)\right)-F_{i, t} \cosh \left(R_{i}\left(z-\left(a_{i}-l_{i} / 2\right)\right)\right)}{K_{v, i} R_{i} \sinh \left(R_{i} l_{i}\right)} & a_{i}-l_{i} / 2 \leq z \leq a_{i}+l_{i} / 2, \\
& -\frac{1}{p\left(S_{S, i} p+K_{h, i} \alpha^{2}\right)} \frac{2}{2 \pi l_{i}}, & a_{i}+l_{i} / 2<z \leq L_{i} . \\
\widehat{\bar{\phi}}_{i, t}(\alpha, z, p)= & \frac{F_{i, t} \cosh \left(R_{i}\left(z-L_{i}\right)\right)-F_{i+1, b} \cosh \left(R_{i}\left(z-\left(a_{i}+l_{i} / 2\right)\right)\right)}{K_{v, i} R_{i} \sinh \left(R_{i}\left(L_{i}-\left(a_{i}+l_{i} / 2\right)\right)\right)}, &
\end{aligned}
$$

The coefficients $F_{i, b}, F_{i, w}, F_{i, t}$ represent the fluxes at the levels $z=L_{i-1}, a_{i}-l_{i} / 2, a_{i}+l_{i} / 2$, respectively, where $F_{N+1, b}$ is the flux at the upper boundary of the system of $N$ layers. By this representation the continuity conditions with respect to the fluxes (the second condition in (35-37)) have been satisfied automatically. These coefficients can be found by inversion of a tridiagonal, symmetric $(3 N-1,3 N-1)$-matrix, if there is one partially penetrating filter in each aquifer. Otherwise, the dimension of the matrix becomes $\left(N+2 N_{w}-1, N+2 N_{w}-1\right)$, where $N_{w}$ $\left(N_{w} \leq N\right)$ is the number of filters at $r=0$. Using Cramer's rule one may express these coefficients analytically in terms of the parameters of the problem $S_{s, i}, K_{h, i}, K_{v, i}, L_{i}, a_{i}$, and $l_{i}$ and the transform parameters $p$ and $\alpha^{2}$ in a complicated way. The inverse transforms have to be done numerically as was done in [26]. Clearly, the analytical solution thus found belongs also to Class 7, as described in Sect. 4.

\section{Applications}

In this section we apply the solution technique as specified in (27) to three different cases. For the Laplace inversion we employed the Gaver-Stehfest method [22]. For these applications we show how the water particles move under the influence of the wells. This is possible because we can now easily derive analytically the velocity field based on the representation of the groundwater head, using the relations

$v_{r}=-\varepsilon_{i} K_{h, i} \frac{\partial \phi_{i}(r, z, t)}{\partial r}, \quad v_{z}=-\varepsilon_{i} K_{v, i} \frac{\partial \phi_{i}(r, z, t)}{\partial z}, \quad i=1, \ldots, N$,

where $v_{r}, v_{z}\left[\mathrm{LT}^{-1}\right]$ are the velocties in the $r$ - and $z$-directions, respectively, and $\varepsilon_{i}[-]$ is the porosity of layer $i$.

We constructed Matlab scripts for evaluation of the groundwater head (plots as function of $r, z$ or $t$, and contour plots as function of $(r, z),(r, t)$ or $(z, t))$ and for evaluation of the velocity field as function of $(r, z, t)$.

\subsection{Case with 2 layers}

For this case we refer to [28]. Therein, a system was considered consisting of one aquifer and one aquitard, with an impermeable base and a specified head at the top. A condition was derived under which a point extraction caused the path of a water particle moving to and from that extraction to be nonmonotonic. That condition reads

$R=K c / a>4$,

where $K=K_{h, 1}=K_{v, 1}$, the hydraulic conductivity of the aquifer, $c=D_{2} / K_{v, 2}$, the hydraulic resistance of the aquitard, $a=D_{1}-a_{1}$, the distance from the top of the aquifer to the point extraction. 


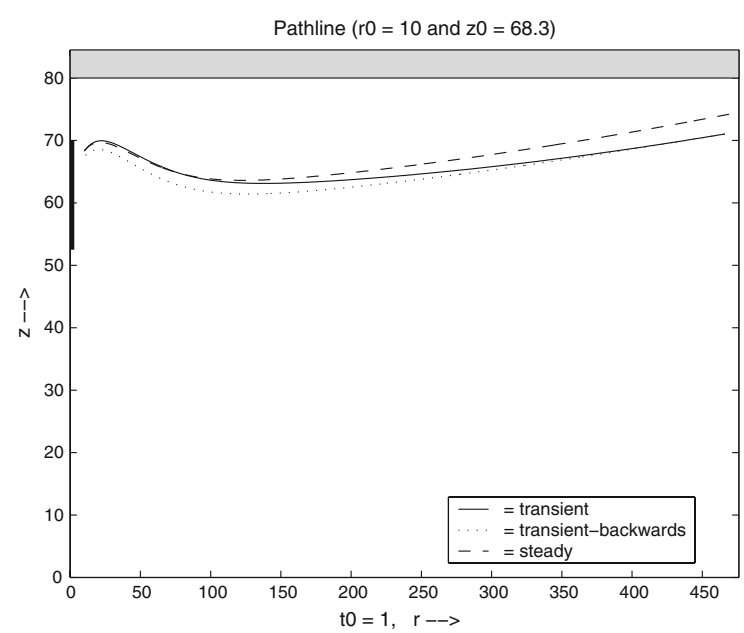

Fig. 1 Transient and steady pathlines for the two-layer case (Sect. 6.1) for particles starting at $r=10[\mathrm{~m}], z=68.3$ [m], at $t=1$ [d]. At $t=2000$ [d] the injection becomes an extraction. The backward flowpath of that particle is also shown

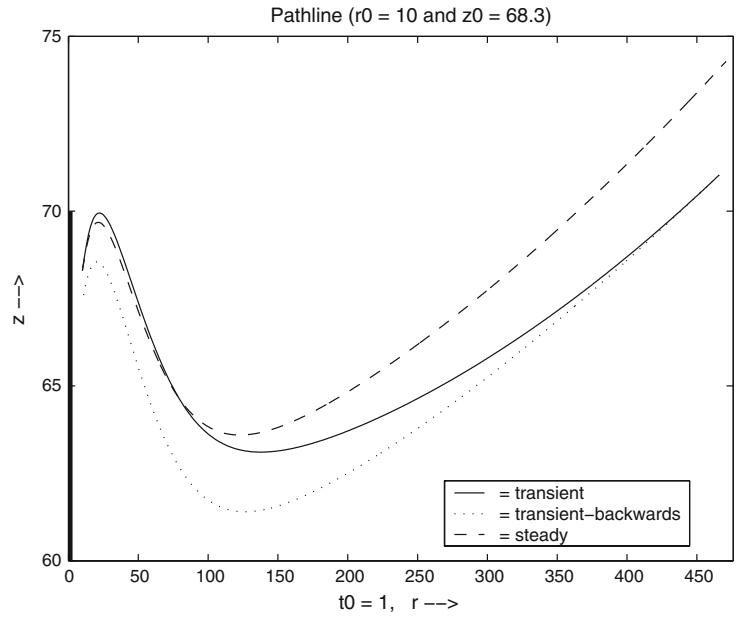

Fig. 2 Enlarged view of Fig. 1

For the current example the layer parameters are:

\begin{tabular}{|c|c|c|c|c|c|c|c|}
\hline Layer & $D[\mathrm{~m}]$ & $K_{h}[\mathrm{~m} / \mathrm{d}]$ & $K_{v}[\mathrm{~m} / \mathrm{d}]$ & $S_{s}[1 / \mathrm{m}]$ & $\varepsilon[-]$ & $\alpha[-]$ & $\beta[\mathrm{T}]$ \\
\hline 1 & 80 & 25 & 25 & 0.05 & 0.3 & $\alpha_{0}=0$ & $\alpha_{0}=1$ \\
\hline \multirow[t]{3}{*}{2} & 4.5 & 0.01 & 0.01 & 0.05 & 0.3 & $\beta_{1}=1$ & $\beta_{1}=0$ \\
\hline & \multirow[t]{2}{*}{ v } & $Q\left[\mathrm{~m}^{3} / \mathrm{d}\right]$ & \multicolumn{2}{|c|}{ Bottom well [m] } & \multicolumn{2}{|c|}{ Top well [m] } & \\
\hline & & -9240 & 52.5 & & 70 & & \\
\hline
\end{tabular}

Condition (43) can be applied if the extraction is considered to be concentrated at the well center $z=a_{1}$. It is found that $R=600$ hence, (43) is satisfied.

We first followed a particle starting from the well $\left(Q_{\text {injection }}=-9240\left[\mathrm{~m}^{3} / \mathrm{d}\right]\right)$ at $r_{0}=10[\mathrm{~m}]$, and $z_{0}=68.3[\mathrm{~m}]$ at time $t_{0}=1[\mathrm{~d}]$ up to $t_{\text {end }}=2000$ [d] under a transient regime (the solid line in Figs. 1,2). Second, we followed another particle from the well by the same injection rate under a steady regime (the dashed line in Figs. 1, 2), and finally, we started an extraction at $t_{\text {end, injection }}=2000[\mathrm{~d}]$ at the same rate $\left(Q_{\text {extraction }}=-Q_{\text {injection }}\right)$ and followed the first particle backwards from the point where it had arrived during the injection up till the starting distance from the well $\left(r_{\text {start }}=10[\mathrm{~m}]\right)$ (the dotted line in Figs. 1, 2). The particle reached that starting distance at a level of $z_{\text {end }}=67.437[\mathrm{~m}]$ at the time $t_{\text {end,extraction }}=3957.51[\mathrm{~d}]$. This means that the point of arrival was $0.863[\mathrm{~m}]$ lower than the starting point and that the backwards journey went faster (in $t_{\text {end,injection }}-t_{\text {end,extraction }}=1957.51$ [d]). The flow path for the steady case ends farther from the well than for the transient case.

\subsection{Case with 4 layers}

Next, we consider a system with 4 layers, two aquifers and two aquitards, with an impermeable base and a specified head at the top. There are two wells active: an injection well in the bottom aquifer and an extraction well in layer 3 with the same rate but opposite sign. The parameters are: 


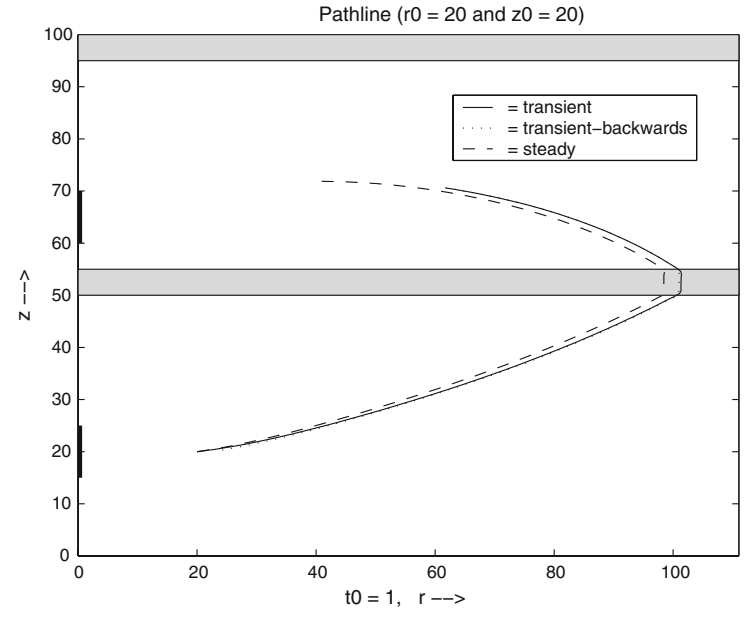

Fig. 3 Transient and steady pathlines for the four-layer case (Sect.6.2) for particles starting at $r=20[\mathrm{~m}], z=20$ [m], at $t=1$ [d]. At $t=191$ [d] the injection becomes an extraction. The backward flowpath is also shown. The path through the aquitard is almost vertical

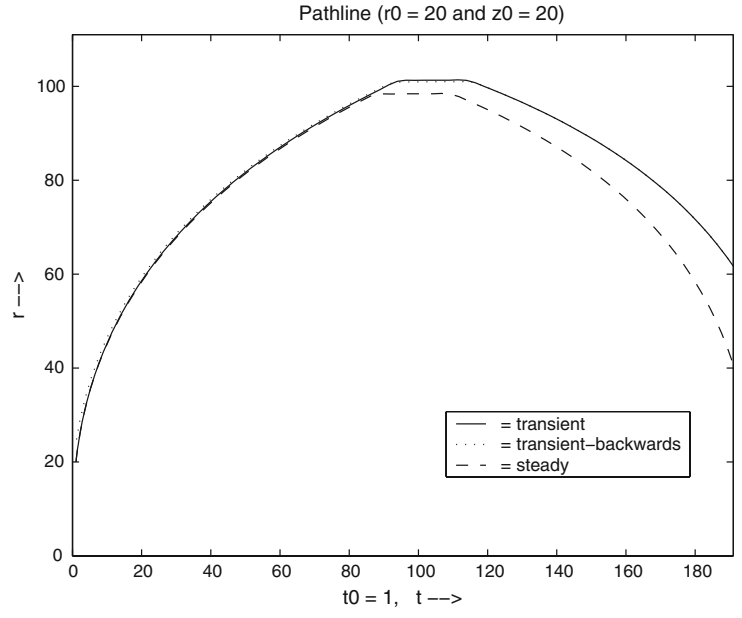

Fig. 4 Transient and steady pathlines for the four layer case (Sect.6.2) for particles starting at $r=20[\mathrm{~m}], z=20[\mathrm{~m}]$, at $t=1[\mathrm{~d}]$. The position of the vertical location $z$ is shown as a function of time $t$. At $t=191$ [d] the injection becomes an extraction. The corresponding flowpath is shown with a reversed time-axis, to show the differences with the forward path. The horizontal sections of the curves belonging to the passage through the aquitard, are clearly visible. That passage time is shorter in the steady case

\begin{tabular}{lrrrllll}
\hline Layer & $D[\mathrm{~m}]$ & $K_{h}[\mathrm{~m} / \mathrm{d}]$ & $K_{v}[\mathrm{~m} / \mathrm{d}]$ & $S_{s}[1 / \mathrm{m}]$ & $\varepsilon[-]$ & $\alpha[-]$ & $\beta[\mathrm{T}]$ \\
\hline 1 & 50 & 25 & 25 & 0.01 & 0.3 & $\alpha_{0}=0$ & $\beta_{0}=1$ \\
2 & 5 & 1 & 1 & 0.05 & 0.3 & - & - \\
3 & 40 & 40 & 40 & 0.1 & 0.3 & - & - \\
4 & 5 & 1 & 1 & 0.1 & 0.3 & $\alpha_{4}=1$ & $\beta_{4}=0$ \\
\hline
\end{tabular}

\begin{tabular}{lllll}
\hline Well & $Q\left[\mathrm{~m}^{3} / \mathrm{d}\right]$ & Layer & Bottom well $[\mathrm{m}]$ & Top well $[\mathrm{m}]$ \\
\hline 1 & -9240 & 1 & 15 & 25 \\
2 & 9240 & 3 & 60 & 70 \\
\hline
\end{tabular}

See Fig. 3 for the path as a function of $r$ and $z$, and Fig. 4 as a function of $t$ and $z$.

\subsection{Case with 6 layers}

Finally, we consider a system of 6 layers, three aquifers and three aquitards, with an impermeable base and top. There are three wells active: injection wells in the bottom aquifer and in the top aquifer and an extraction well in the middle aquifer. The net rate of the injections and extraction is zero. The parameters are:

\begin{tabular}{lrrrllll}
\hline Layer & $D[\mathrm{~m}]$ & $K_{h}[\mathrm{~m} / \mathrm{d}]$ & $K_{v}[\mathrm{~m} / \mathrm{d}]$ & $S_{s}[1 / \mathrm{m}]$ & $\varepsilon[-]$ & $\alpha[-]$ & $\beta[\mathrm{T}]$ \\
\hline 1 & 20 & 25 & 25 & 0.01 & 0.3 & $\alpha_{0}=0$ & $\beta_{0}=1$ \\
2 & 5 & 5 & 1 & 0.05 & 0.3 & - & - \\
3 & 20 & 25 & 25 & 0.1 & 0.3 & - & - \\
4 & 5 & 5 & 1 & 0.1 & 0.3 & - & - \\
5 & 20 & 25 & 25 & 0.1 & 0.3 & - & - \\
6 & 5 & 5 & 5 & 0.1 & 0.3 & $\alpha_{6}=0$ & $\beta_{6}=1$ \\
\hline
\end{tabular}




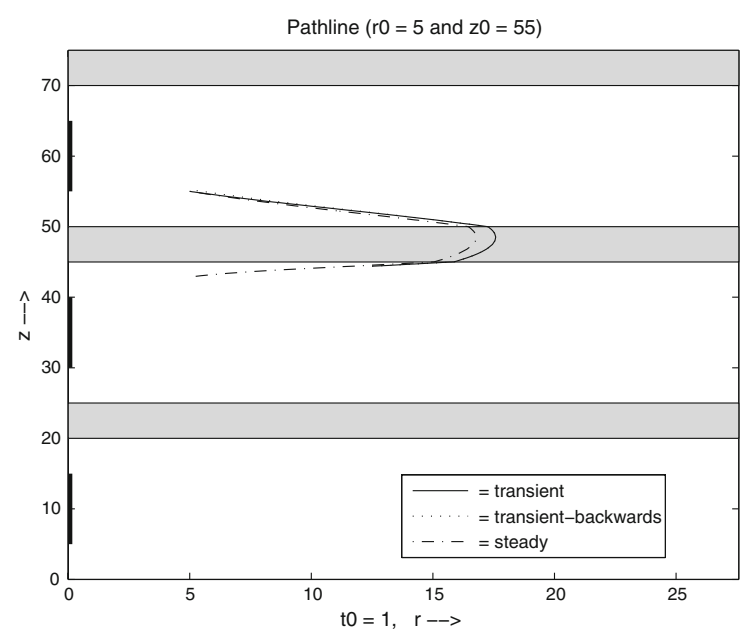

Fig. 5 Transient and steady pathlines for the six layer case (Sect. 6.3) for particles starting at $r=5[\mathrm{~m}], z=55[\mathrm{~m}]$, at $t=1[\mathrm{~d}]$. At $t=38$ [d] the injection becomes an extraction. The backward flowpath is also shown. The influence of the extraction in the middle aquifer is felt more directly in the steady case, because the particle comes closer to that extraction after 37 days

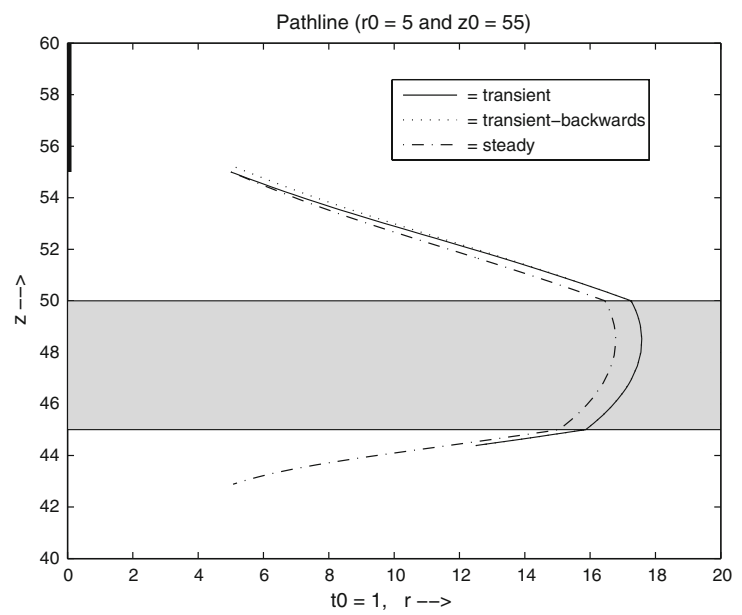

Fig. 6 Enlarged view of Fig. 5

\begin{tabular}{lllll}
\hline Well & $Q\left[\mathrm{~m}^{3} / \mathrm{d}\right]$ & Layer & Bottom well $[\mathrm{m}]$ & Top well $[\mathrm{m}]$ \\
\hline 1 & -500 & 1 & 5 & 15 \\
2 & 1000 & 3 & 30 & 40 \\
3 & -500 & 5 & 55 & 60 \\
\hline
\end{tabular}

See Fig. 5 shows the path as function of $r$ and $z$, and Fig. 6 shows the same for an enlarged view.

\section{Conclusions}

We have applied a generalized Fourier transform, specified by (12-16), and (21) to solve semi-analytically a longstanding problem in hydrology: three-dimensional transient flow in a coupled system of aquifers with $z$-dependence.

This generalized Fourier transform, with the restriction $d_{i}=0$ in (13), has been applied succesfully in the past for cases where one has to deal only with just two independent variables ( $r$ and $z$ (a stationary case); see [1]. It could be applied for the case with the independent variables $t$ and $z$ (an one-dimensional (vertical), transient case), and also in cases where one can justify the assumption that $K_{h, i} / S_{s, i}=\rho$ is independent of $i$. In all those cases the solution will belong to Class 3 of a classification of analytical solutions we proposed in Sect. 4 .

Here, we have relaxed that restriction (so $d_{i} \neq 0$ ) and are able to treat more complicated partial differential equations. We presented two forms of the semi-analytical solution for the full transient solution of the flow in a coupled system, namely (27) and (31), belonging to Class 6, and one form (39) belonging to Class 7 of the proposed classification.

In the first case (27), a numerical Laplace-inversion method has to be applied which uses only real values for the transform parameter $p$. Alternatively, one could use the form (31) and apply the Hankel-inversion integral (11). This method might give rise to numerical complications due to the oscillating character of the Bessel $J_{0}$-function. It is clear that the representation belonging to Class 7 is not very economical with respect to calculation time. For the examples we employed the first case based on the numerical Laplace inversion using the Gaver-Stehfest method [22]. 
As an extension we remark that this generalized Fourier transform can be applied also to cases with an unconfined upper aquifer. Standard approximations (see [29] and e.g. [27]) arrive at a model where the boundary condition at the top (3) has been modified into

$$
K_{v, N} \frac{\partial \phi_{N}}{\partial z}\left(r, L_{N}, t\right)=-S_{Y} \frac{\partial \phi_{N}}{\partial t}\left(r, L_{N}, t\right), \quad t \geq 0, \quad r>0
$$

where $S_{Y}[-]$ is the specific yield. In such cases, we take $\beta_{N}=1 / p$, and $\alpha_{N}=S_{Y}$, with $p$ the Laplace-transform parameter. This will be studied in a future paper.

Finally, the theory presented here is not restricted to groundwater flow, but applies to every layered system of variables which are described by the defining partial differential equation (1). The generalized Fourier transform technique is applicable also in cases where another horizontal spatial operator is active (e.g. $\frac{\partial^{2}}{\partial x^{2}}$ or $\frac{\partial^{2}}{\partial y^{2}}$ or $\left.\left(\frac{\partial^{2}}{\partial x^{2}}+\frac{\partial^{2}}{\partial y^{2}}\right)\right)$.

Open Access This article is distributed under the terms of the Creative Commons Attribution Noncommercial License which permits any noncommercial use, distribution, and reproduction in any medium, provided the original author(s) and source are credited.

\section{Appendix A: Integral transform in terms of $t$ and $z$ for the case $K_{h, i} / S_{s, i}=\rho$}

In this case the remaining problem becomes

$$
p \mathrm{Z}[\bar{\phi}](m)=\rho\left(\frac{\mathrm{d}^{2} \mathrm{Z}[\bar{\phi}](m)}{\mathrm{d} r^{2}}+\frac{1}{r} \frac{\mathrm{dZ}[\bar{\phi}](m)}{\mathrm{d} r}\right)-\lambda_{m}^{2} \mathrm{Z}[\bar{\phi}](m), \quad r>0, \quad m=1, \ldots,
$$

$\lim _{r \rightarrow 0}\left(r \frac{\mathrm{dZ}[\bar{\phi}](m)}{\mathrm{d} r}\right)=\frac{1}{\rho p} \widetilde{Q}(m)$, with $\widetilde{Q}(m)=\sum_{i=1}^{N} \int_{L_{i-1}}^{L_{i}} \frac{Q_{i}}{2 \pi l_{i}} \mathrm{I}_{A_{i}}(z) \psi_{i}^{m}(z) \mathrm{d} z$,

and where

$$
\begin{aligned}
& \mathrm{I}_{A_{i}}(z)=1, \quad a_{i}-l_{i} / 2 \leq z \leq a_{i}+l_{i} / 2, \\
& \mathrm{I}_{A_{i}}(z)=0, \quad L_{i-1} \leq z<a_{i}-l_{i} / 2 \text { and } a_{i}+l_{i} / 2<z \leq L_{i} .
\end{aligned}
$$

In this case $\lambda_{m}$ is independent of $p$. The solution of (A1) now reads

$$
\mathrm{Z}[\bar{\phi}](m)=-\frac{1}{\rho p} \widetilde{Q}(m) \mathrm{K}_{0}\left(r \sqrt{\frac{p+\lambda_{m}^{2}}{\rho}}\right),
$$

where the dependence on $p$ is explicit. The $\lambda_{m}$ values are now independent of $p$ and $\alpha^{2}$. The inverse transform with respect to $p$ gives

$\mathrm{Z}[\phi](m)=-\frac{1}{\rho} \widetilde{Q}(m) \int_{0}^{t} \frac{1}{2 \tau} \exp \left(-\lambda_{m}^{2} \tau-r^{2} /(4 \rho \tau)\right) \mathrm{d} \tau$,

[30, Eq. 29.3.120]. The integral is the well-known Hantush well function, defined as

$$
W(u, w)=\int_{0}^{u} x^{-1} \exp \left(-x-w^{2} /(4 x)\right) \mathrm{d} x
$$

so,

$\mathrm{Z}[\phi](m)=-\frac{1}{\rho} \widetilde{Q}(m) W\left(\lambda_{m}^{2} t, \sqrt{\frac{\lambda_{m}^{2} r^{2}}{\rho}}\right) / 2$. 
Clearly, this solution belongs to Class 3, as described in Sect.4. This result can also be written in terms of the so-called generalized incomplete Gamma function $\gamma(\alpha, x ; b)[31,(2.64)]$ defined as

$\gamma(\alpha, x ; b)=\int_{0}^{x} t^{\alpha-1} \exp \left(-t-b t^{-1}\right) \mathrm{d} t$,

namely

$\mathrm{Z}[\phi](m)=-\frac{1}{\rho} \widetilde{Q}(m) \gamma\left(0, \lambda_{m}^{2} t ; \frac{\lambda_{m}^{2} r^{2}}{4 \rho}\right) / 2$,

because $W(u, w)=\gamma\left(0, u ; w^{2} / 4\right)$. As a curiosity we remark that $\gamma(\alpha, x ; b)$ may be written as a double sum [31, compare Eq. 2.128].

\section{Appendix B: Integral transform in terms of $r$ and $z$ for the case $K_{h, i} / S_{s, i}=\rho$}

In this case (28) becomes after the generalized Fourier transform (with $w_{i}=S_{s, i}$ and $d_{i}=0$ )

$\frac{\mathrm{dZ}[\widehat{\phi}](m)}{\mathrm{d} t}=-\rho \alpha^{2} \mathrm{Z}[\widehat{\phi}](m)-\widetilde{Q}(m)-\lambda_{m}^{2} \mathrm{Z}[\widehat{\phi}](m), \quad t>0, \quad m=1, \ldots$,

where $\widetilde{Q}(m)$ has been defined in (25),

$\mathrm{Z}[\widehat{\phi}](m)=0, \quad t=0$.

The solution of (B1.2) reads

$\mathrm{Z}[\widehat{\phi}](m)=-\widetilde{Q}(m)\left(\frac{1-\exp \left(-\left(\rho \alpha^{2}+\lambda_{m}^{2}\right) t\right)}{\rho \alpha^{2}+\lambda_{m}^{2}}\right)$,

and (by differentiation of this expression with $t$, application of the inverse Hankel transform (11), using [30, Eq. 29.3.75] and integration by $\tau$ )

$\mathrm{Z}[\phi](m)=-\frac{1}{\rho} \widetilde{Q}(m) \int_{0}^{t} \frac{1}{2 \tau} \exp \left(-\lambda_{m}^{2} \tau-r^{2} /(4 \rho \tau)\right) \mathrm{d} \tau$.

Of course, (B4) and (A3) are equal.

\section{Appendix C: Integral transform in terms of $t$ and $r$ for the case $K_{h, i} / S_{s, i}=\rho$}

Now, with $w_{i}=S_{s, i}, d_{i}=0$, the equivalent of (38) becomes

$p \mathrm{Z}[\widehat{\bar{\phi}}](m)=-\rho \alpha^{2} \mathrm{Z}[\widehat{\widehat{\phi}}](m)-\frac{1}{p} \widetilde{Q}(m)-\lambda_{m}^{2} \mathrm{Z}[\widehat{\bar{\phi}}](m), \quad t>0, \quad m=1, \ldots$,

with the solution

$\mathrm{Z}[\widehat{\bar{\phi}}](m)=-\frac{\widetilde{Q}(m)}{p\left(p+\rho \alpha^{2}+\lambda_{m}^{2}\right)}$.

In this case $\lambda_{m}$ is independent of $p$ and $\alpha^{2}$, and so $N_{m}$. The inverse transform with repect to $p, \mathrm{Z}[\widehat{\phi}]$ ( $m$ ), delivers (B3), so, again $\mathrm{Z}[\phi](m)$ is found as (A3) or (B4). Here the generalized Fourier transform with corresponding eigenvalues has to be calculated only once, and depends just on the parameters of the problem $S_{s, i}, K_{h, i}=S_{s, i} \rho$, $K_{v, i}$, and $L_{i}$. 


\section{References}

1. Maas C (1987) Groundwater flow to a well in a layered porous medium 1. Steady flow. Water Resour Res 23(8):1675-1681

2. Veling EJM (1991) FLOP3N_pathlines in three-dimensional groundwater flow in a system of homogeneous anisotropic layers. Technical report Report nr. 719106001, R.I.V.M., National Institute of Public Health and Environmental Protection, Bilthoven, The Netherlands. http://www.citg.tudelft.nl/live/pagina.jsp?id=4f94d86a-e4eb-41b2-af1b-3441ed059de2\&lang=en\&binary=/ doc/doc-f3n-t.pdf

3. Veling EJM (1992) Three-dimensional groundwaterflow modelling for the calculation of capture zones around extraction sites. In: Hirsch Ch, Périaux J, Kordulla W (eds) Computational fluid dynamics, vol 2. In Proceedings of the first European computational fluid dynamics conference, September 7-11, 1992, Brussel, Elsevier, Amsterdam, pp 1013-1020

4. Neuman SP, Witherspoon PA (1969) Theory of flow in a confined two aquifer system. Water Resour Res 5(4):803-816

5. Hemker CJ, Maas C (1987) Unsteady flow to wells in layered and fissured aquifer systems. J Hydrol 90:231-249

6. Maas C (1987) Groundwater flow to a well in a layered porous medium 2. Nonsteady multiple-aquifer flow. Water Resour Res 23(8):1683-1688

7. Senda K, Tuzuki M (1966) Integration von anomalen linearen Anfangsrandwertaufgaben. Technol Rep Osaka Univ 16(689): 89-120

8. Senda K (1968) A family of integral transforms and some applications to physical problems. Technol Rep Osaka Univ 18(823): 261-286

9. Ölçer NY (1968) Theory of unsteady heat conduction in multicomponent finite regions. Ing Arch 36(5):285-293

10. Özişik MN (1980) Heat conduction. Wiley, New York

11. Mikhailov MD, Özişik MN (1984) Unified analysis and solutions of heat and mass diffusion. Dover Publications, Inc., New York

12. Maas C (1986) The use of matrix differential calculus in problems of multiple-aquifer flow. J Hydrol 88:43-67

13. Hemker CJ (1984) Steady groundwater flow in leaky multiple-aquifer systems. J Hydrol 72:355-374

14. Hemker CJ (1985) Transient well flow in multiple-aquifer systems. J Hydrol 81:111-126

15. Hemker CJ (1999a) Transient flow in vertically heterogeneous aquifers. J Hydrol 225:1-18

16. Hemker CJ (1999b) Transient well flow in layered aquifer systems: the uniform well-face drawdown solution. J Hydrol 225:19-44

17. Bruggeman GA (1999) Analytical solutions of geohydrological problems. Developments in Water Science, nr. 46. Elsevier, Amsterdam

18. Sneddon IN (1972) The use of integral transforms. McGraw-Hill, New York

19. Mikhailov MD, Vulchanov NL (1983) Computational Procedure for Sturm-Liouville problems. J Comput Phys 50:323-336

20. Zettl A (2005) Sturm-Liouville theory. Mathematical surveys and monographs, vol 121. American Mathematical Society, Providence

21. Wittrick WH, Williams FW (1971) A general algorithm for computing natural frequencies of elastic structures. Q J Mech Appl Math 24(1):263-284

22. Stehfest H (1970) Algorithm 368, numerical inversion of Laplace transforms. Commun ACM 13(1,10):47-49 and 624

23. de Hoog FR, Knight JH, Stokes AN (1982) An improved method for numerical inversion of Laplace transforms. SIAM J Sci Stat Comput 3(3):357-366

24. Strack ODL (1989) Groundwater mechanics. Prentice Hall, Englewood Cliffs. Out of print, currently available by Strack Consulting Inc., St. Paul

25. Furman A, Neuman SP (2003) Laplace-transform analytic element solution of transient flow in porous media. Adv Water Resour 26:1229-1237

26. Lenoach B, Ramakrishnan TS, Thambynayagam RKM (2004) Transient flow of a compressible fluid in a connected layered permeable medium. Trans Porous Media 57:153-169

27. Malama B, Kuhlman KL, Barrash W (2007) Semi-analytical solution for flow in leaky unconfined aquifer-aquitard systems. J Hydrol 346:59-68. doi:101016/jjhydrol200708018

28. Bruggeman GA, Veling EJM (2006) Nonmonotonic trajectories to a partially penetrating well in a semiconfined aquifer. Water Resour Res 42(2):W02501. doi:101029/2005WR003951

29. Neuman SP (1972) Theory of flow in unconfined aquifers considering delayed response of the water table. Water Resour Res 8(4):1031-1045

30. Abramowitz M, Stegun IA (eds) (1964) Handbook of mathematical functions. National Bureau of Standards, Washington, DC. http://www.math.hkbu.edu.hk/support/aands/tochtm

31. Chaudhry AM, Zubair SM (2002) On a class of incomplete gamma functions with applications. Chapman \& Hall/CRC, Boca Raton 